\title{
Las tecnologías de bajo impacto como alternativa para aprovechar el agua de lluvia en viviendas unifamiliares
}

\author{
Liliana Lizárraga-Mendiola ${ }^{1 \mathrm{a}}$, Carlos Alfredo Bigurra-Alzati ${ }^{2 b, *}$, Francisco Omar Lagarda-García ${ }^{\mathrm{b}}$, Silvia Montiel-Palma ${ }^{\mathrm{a}}$, \\ María del Refugio González-Sandoval ${ }^{\mathrm{a}}$ \\ ${ }^{a}$ CA Ingeniería Civil Sustentable y Tecnología de Materiales, Universidad Autónoma del Estado de Hidalgo. Carr. Pachuca-Tulancingo km. 4.5, Col. Carboneras. \\ C.P. 42184, Mineral de la Reforma, Hidalgo, México. \\ ${ }^{b}$ GI Arquitectura, Tecnología y Habitabilidad. Universidad Autónoma del Estado de Hidalgo. Carr. Pachuca-Tulancingo km. 4.5, Col. Carboneras. C.P. 42184, \\ Mineral de la Reforma, Hidalgo, México. \\ *Autor de correspondencia: carlos.a.bigurra.alzati@gmail.com
}

\begin{abstract}
Resumen
En este trabajo se analizaron las posibilidades de implementar tecnologías de bajo impacto (cosecha de agua de lluvia en azoteas y pavimento permeable) en viviendas de tipo unifamiliar. Se cuantificaron los volúmenes de agua de lluvia cosechables y se diseñó una mezcla de pavimento permeable para determinar sus propiedades mecánicas (resistencia a la compresión) e hidráulicas (permeabilidad). Se observó que es posible cosechar volúmenes suficientes de agua en azoteas de viviendas unifamiliares con superficies de $100 \mathrm{~m}^{2}$ que satisfagan al menos el $25 \%$ del volumen necesario para fines no potables. Además, es posible facilitar la infiltración de escorrentía superficial a través de pavimentos permeables destinados a estacionamientos, cocheras y andadores. Este tipo de tecnologías podrían auto-construirse por usuarios y propietarios.
\end{abstract}

Palabras Clave: cosecha de agua de lluvia, pavimento permeable, tecnologías de bajo impacto, vivienda unifamiliar.

\section{Introducción}

El rápido crecimiento en zonas urbanas reduce las áreas que anteriormente favorecían la interceptación del agua de lluvia en superficie o la infiltración y recarga de acuíferos. Las ciudades de Pachuca de Soto y Mineral de la Reforma no son la excepción: muchas zonas dispersas en las partes bajas de ambas ciudades presentan encharcamientos durante la época de lluvias, lo que ocasiona principalmente congestionamientos viales, daño al pavimento, así como un desaprovechamiento de áreas verdes y superficies que podrían volver a favorecer su interceptación e infiltración. Esta problemática podría reducirse si el agua de lluvia se cosecha a nivel de azoteas, ya que reduciría el volumen que escurre sobre vialidades. Además, si se implementa el pavimento permeable al menos en estacionamientos, cocheras y andadores, ese volumen escurrido podría infiltrarse hacia el subsuelo y evitar su conducción hacia el drenaje público.

Cuando las ciudades no cuentan con sistemas eficientes para la recolección del agua de lluvia, ésta se mezcla con el drenaje urbano municipal, el cual transporta el agua residual proveniente de las casas, comercios y otro tipo de construcciones predominantes en una ciudad (Ahiablame et al., 2013; Angrill et al., 2017).
Por el contrario, las tecnologías de bajo impacto (por sus siglas en inglés "Low Impact Development", LID) son una filosofía que se enfoca en la rehabilitación de ciclos hidrológicos urbanos. Las tecnologías LID se orientan hacia el diseño descentralizado de infraestructura que sirva para aprovechar de una manera equilibrada la escorrentía superficial urbana, alterando lo menos posible las condiciones hidrológicas existentes previas a la urbanización de una ciudad (Davis, 2008). Entre las tecnologías LID más comunes están el pavimento permeable y la cosecha de agua de lluvia a través de las azoteas. La incorporación de este tipo de sistemas en las ciudades facilita la evapotranspiración, infiltración y recarga de acuíferos, así como su recuperación para usos no potables a nivel doméstico.

Sistemas ingenieriles-ecológicos tales como los LID, son prácticas que se caracterizan por ser auto-sostenidos, lo que implica que sólo la infraestructura inicial es responsabilidad del ser humano, mientras que su supervivencia y permanencia en un entorno urbano deben funcionar por sí mismas. Este trabajo describe cómo estas tecnologías se pueden implementar a nivel de vivienda unifamiliar y ser autoconstruidas por el propietario y usuarios. Por un lado, se cuantificó teóricamente el volumen de agua de lluvia cosechable en azoteas de viviendas unifamiliares con fines de almacenamiento y reutilización para uso doméstico no potable.

\footnotetext{
${ }^{1}$ Liliana Lizárraga-Mendiola

* Carlos Alfredo Bigurra-Alzati

Correos electrónicos: lililga.lm@gmail.com; carlos.a.bigurra.alzati@gmail.com; arqlagarda@yahoo.com; silvia_montiel@uaeh.edu.mx; cuquisglezs@gmail.com
} 
Por otro lado, se diseñó una mezcla de pavimento permeable con resistencia a la compresión y permeabilidad aptas para instalarse en andadores, estacionamientos y cocheras. Como vivienda unifamiliar se entiende "habitación que da alojamiento a una familia y que está construida en un terreno propio e independiente" (CONAVI, 2010).

\section{Materiales y Métodos}

\subsection{Cosecha de Agua de Lluvia}

Para conocer el volumen de almacenamiento, es necesario considerar los valores promedio mensuales de precipitación ( $\mathrm{Pi}, \mathrm{mm}$ ) para un periodo mínimo de diez años. Con base en un estudio previo (Lizárraga-Mendiola et al., 2015), se tomaron como base los datos siguientes para el año 2010, considerado como el año más lluvioso en un periodo histórico de 33 años (1980-2013) (Tabla 1):

Tabla 1. Precipitación mensual para el año más lluvioso (2010)

\begin{tabular}{ccccccccccccc}
\hline E & F & M & A & M & J & J & A & S & O & N & D & Prom. \\
\hline 38.2 & 84.6 & 0.0 & 64.3 & 6.7 & 48.8 & 249.4 & 31.7 & 49.1 & 12.8 & 0.0 & 0.0 & 48.8 \\
\hline \multicolumn{1}{c}{ Prom. = Promedio }
\end{tabular}

El número de habitantes por cada vivienda $(\mathrm{Nd})$ es de 3.7 (INEGI, 2016). Para este trabajo, se consideró una dotación necesaria (Dot) por habitante por día de $125 \mathrm{~L}$, basada en el suministro municipal; superficies de azotea de concreto (INEGI, 2016), con un coeficiente de escurrimiento (C, adimensional) de 0.8 y un área de captación promedio (Ac) de $100 \mathrm{~m}^{2}$. A través de la ecuación 1 , se calculó la demanda total $\left(\mathrm{Dt}, \mathrm{m}^{3}\right)$ :

$$
D t=\frac{(N u)(N d)(D o t)}{1000}
$$

donde:

$\mathrm{Nu}=$ número de días del mes analizado.

$\mathrm{Nd}=$ número de usuarios.

Dot $=$ dotación necesaria $(\mathrm{L} / \mathrm{hab} / \mathrm{d})$.

La oferta total equivale al volumen cosechable $\left(\mathrm{Ai}, \mathrm{m}^{3}\right)$, según la ecuación 2:

$$
A i=\frac{(P i)(C)(A c)}{1000}
$$

Después, se estimó el volumen de almacenamiento (Ecuación 3):

$$
\begin{aligned}
& V i=A a i-D a i \\
& \text { donde: }
\end{aligned}
$$

$\mathrm{Vi}=$ volumen de almacenamiento $\left(\mathrm{m}^{3}\right)$.

Aai $=$ oferta mensual acumulada $\left(\mathrm{m}^{3}\right)$.

Dai= demanda mensual acumulada $\left(\mathrm{m}^{3}\right)$.

\subsection{Pavimento permeable}

Para diseñar el pavimento permeable se consideró el tamaño y proporción de agregados, tal como se indica en la siguiente Tabla 2:

Tabla 2: Granulometría del material empleado para elaborar los especímenes

\begin{tabular}{lllc}
\hline $\mathrm{N}^{\circ}$ malla & \multirow{2}{*}{$\begin{array}{l}\text { Tipo de } \\
\text { material }\end{array}$} & \multicolumn{2}{c}{ Cantidad de material } \\
\cline { 3 - 4 } & kg & $\%$ \\
\hline $1 / 2 ”$ & Grava & 2.432 & 40 \\
\hline
\end{tabular}

\begin{tabular}{llll}
\hline 3/8” & Grava & 1.824 & 30 \\
\hline $\mathbf{1 / 4 ”}$ & Grava & 1.216 & 20 \\
\hline $\mathbf{\# 4}$ & Grava & 0.608 & 10 \\
\hline & Arena & 0.608 & 10 \\
\hline
\end{tabular}

Después de cribar el material en las cantidades y tamaños de partícula a utilizar, se elaboraron por duplicado los especímenes de concreto o pavimento permeable (DOF, 2016). Las proporciones utilizadas para elaborar $1 \mathrm{~m}^{3}$ de pavimento permeable se muestran en la Tabla 3:

Tabla 3: Proporción de material usado para elaborar $1 \mathrm{~m}^{3}$ de pavimento permeable

\begin{tabular}{lll}
\hline Material & Proporción & Unidad \\
\hline Cemento & 350 & $\mathrm{~kg} / \mathrm{m}^{3}$ \\
\hline Arena & 127.5 & $\mathrm{~kg} / \mathrm{m}^{3}$ \\
\hline Grava & 1275 & $\mathrm{~kg} / \mathrm{m}^{3}$ \\
\hline Agua & 140 & $\mathrm{~L} / \mathrm{m}^{3}$ \\
\hline
\end{tabular}

A los 28 días de la elaboración y curado de los especímenes, se realizaron pruebas en laboratorio para medir su resistencia a la compresión (DOF, 2015). Para determinar la permeabilidad del pavimento se vertieron $15 \mathrm{~L}$ de agua de manera que no escurriera por los lados del espécimen de concreto. Se recolectó el agua que se filtraba en un recipiente, y se midió el tiempo que tardaba en atravesar el material. Esto se repitió diez veces para obtener un promedio de la permeabilidad.

\section{Resultados y Discusión}

\subsection{Sustitución de agua de lluvia cosechada para fines no potables}

La demanda total (Dt) requerida en una vivienda unifamiliar varía a través del año únicamente debido al número de días del mes correspondiente (Figura 1). Por lo tanto, el mes con menor demanda es febrero, mientras que 7 meses del año requieren un volumen cercano a $14.5 \mathrm{~m}^{3}$ y 5 meses a $14 \mathrm{~m}^{3}$. Aquí debe tomarse en cuenta que hay días y periodos del año en que los consumos diarios aumentan o disminuyen (periodo vacacional, fines de semana, entre otros factores).

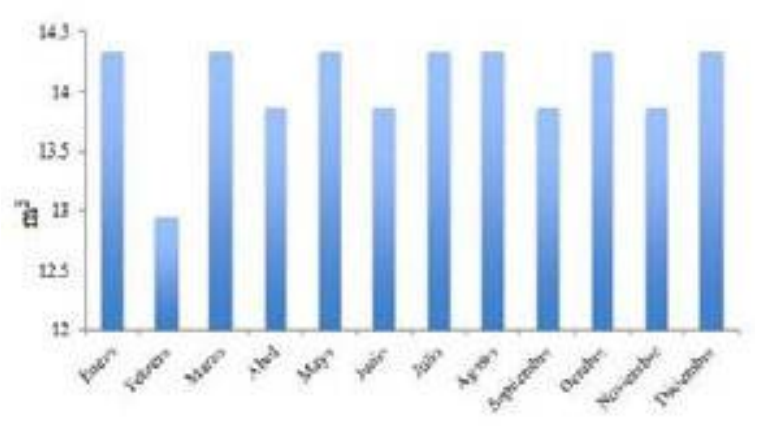

Figura 1: Demanda de agua potable total requerida por vivienda unifamiliar 
Por otra parte, la capacidad de viviendas unifamiliares de $100 \mathrm{~m}^{2}$ de superficie de azotea para cosechar un volumen suficiente (Ai) que satisfaga las necesidades de consumo no potable durante todo el año, son bajas (Figura 2).

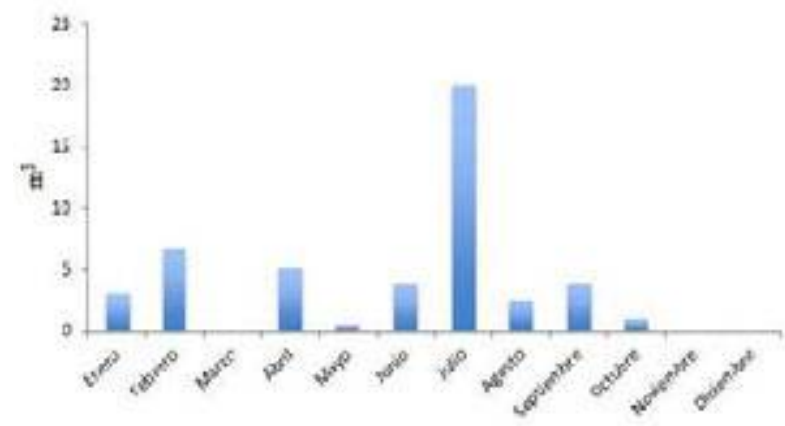

Figura 2: Volúmenes de agua de lluvia cosechables en azotea de vivienda unifamiliar

Esto se debe a que los volúmenes cosechables sólo son eficientes durante los meses de lluvias (abril a octubre). Sin embargo, excepto durante los meses de marzo, noviembre y diciembre, el resto del año sería posible interceptar volúmenes suficientes para cubrir necesidades básicas como limpieza y riego de áreas verdes (alrededor del 25\% del consumo necesario). En cuanto al volumen disponible para almacenamiento, sería insuficiente para satisfacer la Dt de la vivienda unifamiliar (Figura 3).

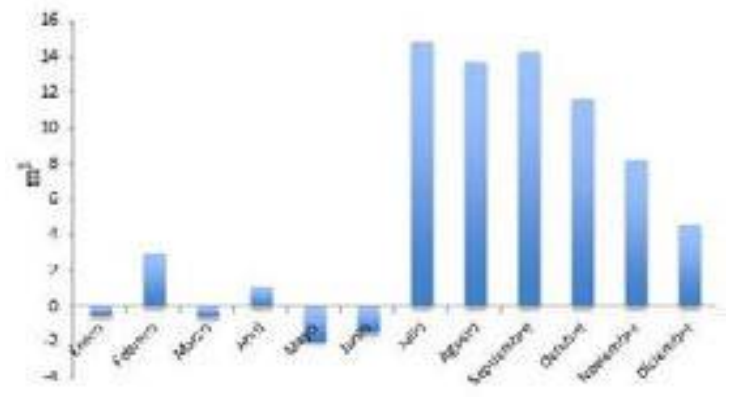

Figura 3: Volúmenes disponibles para fines no potables

Sin embargo, en este trabajo se sugiere utilizar sólo el $25 \%$ del Vi para fines no potables. De acuerdo con esta propuesta, se observa que, excepto enero, marzo, mayo y junio, el resto del año podría sustituirse ese volumen con agua de lluvia cosechada.

\subsection{Capacidad de infiltración en estacionamientos, cocheras y andadores}

En la siguiente figura se pueden observar los resultados de la resistencia a la compresión en especímenes de pavimento permeable (Figura 4).

Los valores obtenidos (máximo de $62.71 \mathrm{~kg} / \mathrm{cm}^{2}$ ) indican que éste puede ser utilizado en andadores, estacionamientos y cocheras. En cuanto a su permeabilidad, las lecturas incluidas en la Tabla 4 permitieron estimar la capacidad de infiltración, donde, para un pavimento permeable con espesor de $7 \mathrm{~cm}$, su permeabilidad es de $21.7 \mathrm{~L} / \mathrm{min}$.

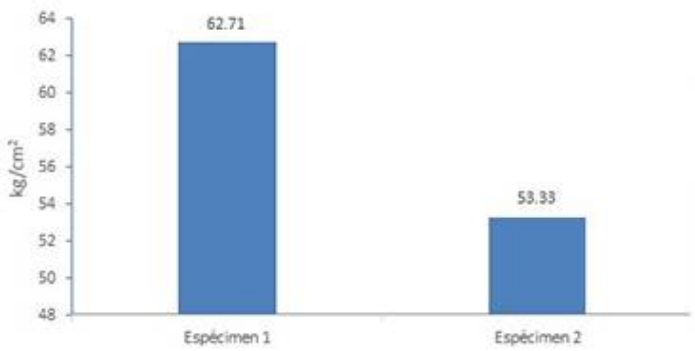

Figura 4. Comportamiento mecánico de los especímenes de concreto permeable

Tabla 4. Lecturas de permeabilidad en especímenes de concreto permeable

\begin{tabular}{lll}
\hline No. de prueba & Cantidad de agua (L) & $\begin{array}{l}\text { Tiempo } \\
(\mathrm{s})\end{array}$ \\
\hline 1 & 15 & 44 \\
\hline 2 & 15 & 47 \\
\hline 3 & 15 & 39 \\
\hline 4 & 15 & 42 \\
\hline 5 & 15 & 39 \\
\hline 6 & 15 & 40 \\
\hline 7 & 15 & 41 \\
\hline 8 & 15 & 40 \\
\hline 9 & 15 & 41 \\
\hline 10 & 15 & 40 \\
\hline Promedio $=$ & & $41.3 \mathrm{~s}$ \\
\hline
\end{tabular}

De acuerdo con datos del IMCYC (2011), aunque la capacidad de drenaje de un pavimento permeable depende del tamaño del agregado, ésta varía entre 81 y 730 $\mathrm{L} / \mathrm{min} / \mathrm{m}^{2}$, por lo que los resultados obtenidos pueden interpretarse como buenos para favorecer la infiltración hacia el subsuelo.

\section{Conclusiones}

Se concluye que las dos tecnologías LID analizadas en este trabajo representan una buena oportunidad de interceptar el agua en azoteas de viviendas unifamiliares y sustituir la cuarta parte necesaria para consumo no potable. En cuanto al pavimento permeable, representa una buena alternativa para favorecer nuevas áreas de recarga en zonas urbanizadas consideradas como impermeables.

\section{English Summary}

Low-impact techniques as an alternative to take advantage of rainwater in familiar houses 


\section{Abstract}

In this work, we analyzed the possibilities of implementing low-impact technologies (rain water harvesting in roofs and permeable pavement) in a family house type housing. It is quantified by the volumes of harvestable rain water and a mixture of permeable pavement to determine their mechanical properties (resistance to the compression) and hydraulic (permeability). It was noted that it is possible to harvest sufficient volumes of water on roofs of houses with a surface of $100 \mathrm{~m}^{2}$ that meet at least $25 \%$ of the volume needed for non-potable purposes. In addition, it is possible to facilitate the infiltration of surface runoff through permeable pavements for parking lots, and garages. This type of technologies could selfbuilt by owners and users.

Keywords:

Rain water harvesting, permeable pavement, low impact development techniques

\section{Agradecimientos}

El Cuerpo Académico "Ingeniería Civil Sustentable y Tecnología de Materiales" agradece al Programa de Mejoramiento al Profesorado, SEP, por el financiamiento otorgado para el fortalecimiento de Cuerpos Académicos 2016 $-2017$.

El Grupo de Investigación "Arquitectura, tecnología y habitabilidad" agradece al Programa de Mejoramiento al Profesorado PRODEP al proyecto para la incorporación de nuevos Profesores de Tiempo Completo PTC 2016 - 2017.

\section{Referencias}

Ahiablame, L. M., Engel, B. A., y Chaubey, I. (2013) Effectiveness of low impact development practices in two urbanized watersheds: Retroffiting with rain barrel/cistern and porous pavement. Journal of Environmental Management, 119, 351-361.

Angrill, S., Petit-Boix, A., Morales-Pinzón, T., Josa, A., Rieradevall, J., y Gabarrell, X. (2017) Urban rainwater runoff quantity and quality A potential endogenous resource in cities?. Journal of Environmental Management, 189, 14-21.

CONAVI (2010) Comisión Nacional de Vivienda. Código de Edificación de Vivienda. 496 p. [en línea]. https://www.gob.mx/cms/uploads/attachment/file/85460/Codigo_de_E dificacion_de_Vivienda.pdf.

Davis, A. P. (2008) Field performance of bioretention: Hydrology impacts. Journal of Hydrological Engineering, 13(2), 90-95.

DOF (2015) Diario Oficial de la Federación. NMX-C-083-ONNCCE2014, Industria de la construcción-Concreto-Determinación de la resistencia a la compresión de especímenes-Método de ensayo [en línea].

http://www.dof.gob.mx/nota_detalle.php?codigo=5387841\&fecha=06/ $04 / 2015$

DOF (2016) Diario Oficial de la Federación. NMX-C-159-ONNCCE2016, Industria de la construcción-Concreto-Elaboración y curado de especímenes de ensayo [en línea].

http://dof.gob.mx/nota_detalle.php?codigo $=5445530 \&$ fecha $=22 / 07 / 20$ 16.

IMCYC (2011) Instituto Mexicano del Cemento y del Concreto, A.C. Concreto permeable: alternativas sustentables. Editado por: Carlos Aire. Construcción y Tecnología en Concreto, junio 2011, número 03 [en línea]. http://www.imcyc.com/revistacyt/jun11/arttecnologia.htm.

INEGI (2016a) Instituto Nacional de Estadística, Geografía e Informática. Inventario nacional de viviendas [en línea]. http://www.beta.inegi.org.mx/app/mapa/inv/.

Lizárraga-Mendiola, L., Vázquez-Rodríguez, G., Blanco-Piñón, A., Rangel-Martínez, Y., y González-Sandoval, M. R. (2015) Estimating the Rainwater Potential per Household in an Urban Area: Case Study in Central Mexico. Water, 7, 4622-4637. 